\title{
Methodological quality of meta-analyses of single-case experimental studies
}

\author{
Laleh Jamshidi $^{\mathrm{a}, \mathrm{b}, *}$, Mieke Heyvaert ${ }^{\mathrm{a}}$, Lies Declercq ${ }^{\mathrm{a}, \mathrm{b}}$, Belén Fernández-Castilla ${ }^{\mathrm{a}, \mathrm{b}}$, \\ John M. Ferron ${ }^{c}$, Mariola Moeyaert ${ }^{\mathrm{d}}$, S. Natasha Beretvas ${ }^{\mathrm{e}}$, Patrick Onghena ${ }^{\mathrm{a}}$, \\ Wim Van den Noortgate ${ }^{a, b}$ \\ ${ }^{\text {a }}$ Faculty of Psychology and Educational Sciences, KU Leuven, University of Leuven, Belgium \\ b IMEC-ITEC, KU Leuven, University of Leuven, Belgium \\ c University of South Florida, Tampa, Florida, USA \\ d State University of New York, Albany, NY, USA \\ ${ }^{\mathrm{e}}$ University of Texas at Austin, TX, USA
}

\section{A R T I C L E I N F O}

Number of reviews completed is 2 The paper is part of a special issue on Single case experimental studies

Keywords:

Single-case experimental design

Systematic review

Meta-analysis

Methodological quality

R-amstar

\begin{abstract}
A B S T R A C T
Background: Methodological rigor is a fundamental factor in the validity and credibility of the results of a meta-analysis.

Aim: Following an increasing interest in single-case experimental design (SCED) meta-analyses, the current study investigates the methodological quality of SCED meta-analyses.

Methods and procedures: We assessed the methodological quality of 178 SCED meta-analyses published between 1985 and 2015 through the modified Revised-Assessment of Multiple Systematic Reviews (R-AMSTAR) checklist.

Outcomes and results: The main finding of the current review is that the methodological quality of the SCED meta-analyses has increased over time, but is still low according to the R-AMSTAR checklist. A remarkable percentage of the studies (93.80\% of the included SCED meta-analyses) did not even reach the midpoint score (22, on a scale of $0-44)$. The mean and median methodological quality scores were 15.57 and 16, respectively. Relatively high scores were observed for "providing the characteristics of the included studies" and "doing comprehensive literature search". The key areas of deficiency were "reporting an assessment of the likelihood of publication bias" and "using the methods appropriately to combine the findings of studies".

Conclusions and implications: Although the results of the current review reveal that the methodological quality of the SCED meta-analyses has increased over time, still more efforts are needed to improve their methodological quality.
\end{abstract}

\section{What this paper adds?}

Through a comprehensive methodological quality assessment of SCED meta-analyses published between 1985 and 2015 , this paper further clarifies important deficiencies in the validity and credibility of the results of these meta-analyses. Future SCED metaanalysts could avoid methodological flaws in the early stages of their studies by paying more attention to these methodological shortcomings.

\footnotetext{
* Corresponding author at: Faculty of Psychology and Educational Sciences, KU Leuven, Etienne Sabbelaan 53, 8500 Kortrijk, Belgium.

E-mail address: laleh.jamshidi@kuleuven.be (L. Jamshidi).
} 


\section{Introduction}

Systematic reviews (SRs) are an important and invaluable tool to combine the evidence about the effects of an intervention of interest in a systematic way and provide key summaries for clinical decision-making. An SR can include a meta-analysis (MA) that statistically combines the results of the individual studies and provides an overall estimate of the effect size, and possibly studies the moderating effect of study characteristics. SRs with high-ranked methodological standards can provide valid and appropriate conclusions. SRs can even be regarded as the most valid form of research aimed at providing evidence-based recommendations for clinical decision making (Faggion \& Giannakopoulos, 2013; Hall, Lee, \& Zurakowski, 2017; Rotta, Salgado, Silva, Correr, \& Fernandez-Llimos, 2015; Wells, Kolt, Marshall, Hill, \& Bialocerkowski, 2013). However, appropriate and proper systematic data collection and analytical methods should be used in SRs and MAs to reflect the current standards for evidence synthesis and to provide accurate evidence about the literature (Hall et al., 2017; Rice, Shrier, Kloda, Benedetti, \& Thombs, 2016). This study explores the methodological quality of MAs of studies applying a single-case experimental design (SCED). Before discussing the methodology, we describe SCEDs, the meta-analysis of SCED studies and the issue of assessing the quality of MAs.

\subsection{Single-case experimental design (SCED) characteristics and features}

SCEDs are used to evaluate the effect of one or more treatments on one or more individual cases. The case may be a participant or another single entity that forms the research unit, such as a school or a family. This entity is repeatedly observed, over the levels of one or several manipulated independent variables (Onghena, 2005). An SCED is identified by three principal features: (a) data are gathered, analyzed, and interpreted for one or multiple cases (this "case" can be a participant, but also another unit, for example, a classroom); (b) the case(s) is/are observed repeatedly during at least two different conditions that are introduced/withdrawn by the researcher; and (c) outcomes are compared across conditions within a case (Beretvas \& Chung, 2008a; Kratochwill et al., 2010; Moeyaert, Ugille, Ferron, Beretvas, \& Van den Noortgate, 2014a; Rogers \& Graham, 2008; Smith, 2012). This kind of design focuses on evaluating whether there is a causal or functional relationship between introducing a treatment as an independent variable and the change in a dependent variable. Because the cases in SCEDs are observed repeatedly over time, the time variable plays an important role in these designs. In the basic version of this design (AB design), the subject is measured repeatedly, before and during or after the treatment or intervention is introduced. The results of these designs can then be analyzed visually or statistically. There are more complex single case designs such as multiple-baseline designs (MBDs), reversal designs, and alternating designs (Barlow, Nock, \& Hersen, 2009).

As Beretvas and Chung (2008b) pointed out, many outcomes of interest in social sciences have natural growth trends (increases or decreases over time) even without intervention/treatment. When a trend over time is present in temporally ordered data, treatment effect sizes that do not explicitly account for these trends may be under- or overestimating the effect (Campbell, 2004). The possibility of developmental trends is one of the main features of the SCED studies.

Another key feature of SCED data is that it is possible that sequential observations are more similar compared to observations that are more distant from each other because the same random factors may continue to influence the scores on multiple subsequent measurement occasions (Van den Noortgate \& Onghena, 2008). This phenomenon is called serial dependency and it implies that residuals are not independently distributed, an assumption made by common statistical tests and analyses (Owens, 2011; Petit-Bois, Baek, Van den Noortgate, Beretvas, \& Ferron, 2016).

\subsection{SCED meta-analysis methods}

SCEDs have been frequently applied in different disciplines to examine the effects of interventions or treatments (Schlosser, Lee, \& Wendt, 2008; Shadish, 2014a, 2014b; Shadish \& Rindskopf, 2007; Smith, 2012). One of the main issues of SCEDs is their limited generalizability and hence their limited usefulness for the development of evidence-based guidelines because of the small number of cases under investigation. To enhance generalizability, researchers replicate SCEDs across cases, across setting, or across behaviors. Meta-analytic procedures allow researchers to quantitatively synthesize the results of these replications and provide evidence for best practices (Beretvas \& Chung, 2008a; Petit-Bois et al., 2016; Tincani \& De Mers, 2016). Interest in the meta-analysis of SCEDs has increased in the past decade (Shadish, 2014a; Shadish, Hedges, \& Pustejovsky, 2014) and many methodological advancements in the field of meta-analysis were made.

SCED meta-analysts can apply different methods and procedures for statistically analyzing the data from multiple SCED studies such as regression analyses of effect sizes, multilevel analyses of raw data or effect sizes, or the calculation of a simple average, median, or range of effect sizes (Owens, 2011; Petit-Bois et al., 2016).

The simplest method for calculating the overall effect is to combine the calculated effect sizes from individual studies by reporting the simple average, weighted average, median, or the range of effect sizes. Another method for providing an overall overview across studies is vote counting. Borenstein, Hedges, Higgins, and Rothstein (2009) considered this method as the process of counting the number of studies that found statistical significance and the number that do not, and then choosing the 'winner'. An, advantage is that the method is still applicable if studies do not provide enough information to calculate an effect size estimate, but contain the information about the direction and the statistical significance of the results, or just the direction of the results (Bushman \& Wang, 2009).

Combining p-values is another way of aggregating data from multiple studies. Borenstein et al. (2009) proposed this method for researchers who want to work directly with the p-values from each test instead of effect sizes in order to obtain an overall p-value. 
Also for analyzing multiple SCED studies testing the same null hypothesis, the p-values of these individual SCED tests can be combined (Onghena \& Edgington, 2005). Tests based on combining p-values examine the null hypothesis that the effect size is zero in all studies. In other words, if combining p-values yields a statistically significant effect, it would be concluded that the effect is real in at least one of the included studies.

Regression models can be applied to evaluate the relationship between study-level covariates and effect sizes. These techniques are analogous to applying multiple regression in primary studies to assess the relationship between case-level covariates and an outcome, but it is recommended to weight effect sizes according to their precision. This method can be performed using a fixed-effect or a random-effect model. Whereas the former assumes that after correcting for the moderating effects of the predictors, the variation in effect sizes is due to sampling variation only, the latter accounts for possible systematic between-study heterogeneity in effect sizes (Hedges and Olkin, 1983, 1985).

Van den Noortgate and Onghena (2003b) proposed multilevel models for the (meta-)analysis of SCEDs. These models are able to aggregate the results of the SCEDs by combining either raw data or effect sizes. One of the advantages of applying a multilevel model is that it allows taking into account the dependencies in the data by allowing for variation within cases, between cases of the same study, and between studies. The model can include linear and non-linear time trends and can be used to study the evolution of the effect over time. Another important advantage of this approach is its flexibility to explore whether study, treatment, or case characteristics can moderate the treatment effect (Van den Noortgate \& Onghena, 2008). Multilevel models can take into account all measurements from all cases measured within and across different conditions and phases instead of focusing on the average effect (Moeyaert, Ugille, Ferron, Beretvas, \& Van den Noortgate, 2014b). Therefore, this approach can handle various additional complexities such as autocorrelation and heterogeneous variances (Moeyaert et al., 2014b; Van den Noortgate \& Onghena, 2003a, 2003b,2008).

\subsection{Methodological quality assessment of $S R$ and $M A$}

The "Garbage in Garbage out"-metaphor in selecting the primary studies is often used to highlight issues related to poor data collection (Sharif, Janjua-Sharif, Sharif, Ali, \& Ahmed, 2013). However, to make decisions based on valid and strong results, it is important to not only critically appraise primary studies but also to critically appraise SRs (Pieper, Mathes, \& Eikermann, 2014). SRs and MAs with major methodological deficiencies might lead to erroneous and inaccurate conclusions about the evidence, which might mislead decision-making processes (Remschmidt, Wichmann, \& Harder, 2014). Conflicting results from SRs can make readers confused (Wells et al., 2013), and make it more difficult for practitioners and clinicians to make appropriate inferences. Systematically following specific steps can help in providing a well-conducted SR: (1) formulating review questions/objectives, (2) defining inclusion and exclusion criteria, (3) locating studies, (4) selecting eligible studies, (5) appraising study quality, (6) extracting data, (7) analyzing and presenting the results, and (8) discussing and interpreting the results (Egger \& Smith, 2001). A critical appraisal of the literature, including SRs, by systematically considering validity, findings, and relevance of past research to the area of interest provides a means of assessing and interpreting the evidence (Sygouros \& Acar, 2013).

Scientists, practitioners and clinicians should keep in mind that SRs and MAs may considerably vary in methodological quality (Remschmidt et al., 2014), and therefore should critically examine their methodological quality (Rotta et al., 2015; Shea et al., 2007). Methodological quality assessment of SRs and MAs is critical to avoid the effect of potentially misleading information when developing clinical guidelines (Faggion, 2015). Moreover, a critical assessment of the methodological quality of SRs with inconsistent results may help in recognizing the potential causes of heterogeneous results and give more useful guidelines to practitioners and clinicians who are interested in the strengths and limitations of the current evidence before making intervention/treatment decisions (Faggion \& Giannakopoulos, 2013). Unfortunately, for readers not specialized in the particular research domain and in the methodology of conducting high quality SRs, it may be difficult to recognize methodologically sound reviews and valid conclusions (Sharif et al., 2013). Therefore, a validated tool can be used to explore how well the SRs and MAs have been conducted and the extent of confidence researchers can have in the results that are provided and associated inferences listed in the reviews (Faggion \& Giannakopoulos, 2013). Selecting an appropriate critical appraisal tool for SRs is not straightforward (Shea, Dube, \& Mohr, 2001). Several tools, such as the Assessment of Multiple Systematic Reviews (AMSTAR), R (Revised)-AMSTAR, Overview Quality Assessment Questionnaire (OQAQ), and Preferred Reporting Items for Systematic Reviews and Meta-Analysis (PRISMA) have been developed to evaluate the methodological quality of SRs and MAs (Kung et al., 2010; Moher, Liberati, Tetzlaff, Altman, \& The PRISMA Group, 2009; Oxman \& Guyatt, 1991; Shea et al., 2007)

SCEDs have been widely used in different fields to examine intervention effects (Schlosser et al., 2008; Shadish, 2014a, 2014b; Smith, 2012), meta-analytic approaches are applied increasingly to generalize the effectiveness of the intervention and quantitatively aggregate past research results to provide evidence for best practices (Beretvas \& Chung, 2008a; Owens, 2011; Petit-Bois et al., 2016; Tincani \& De Mers, 2016). Again, high quality and valid results play a critical role in making decisions about applying treatments or interventions.

Assessments of the quality of SRs and MAs in different fields such as health sciences (Alarcón et al., 2017; Bao et al., 2014; Corry, While, Neenan, \& Smith, 2015; Deckert, Kopkow, \& Schmitt, 2014; Faggion \& Giannakopoulos, 2013; Kowalczuk, Adamich, Simunovic, Farrokhyar, \& Ayeni, 2015; Li et al., 2012; O’Donnell et al., 2014; Parke et al., 2015; Pearce et al., 2015; Pinnock et al., 2017) and psychology (Brossart, Meythaler, Parker, McNamara, \& Elliott, 2008; Machalicek et al., 2010) indicate some methodological deficiencies in the reviews (e.g., not applying a random effects model in case of heterogeneity, not assessing the likelihood of publication bias, not assessing the scientific quality of included studies in formulating the conclusions, among others) that can limit the validity of their results. As some deficiencies have previously been observed by other researchers in assessing the methodological 
quality of SRs and MAs in different fields, it can be expected that similar issues occur in SCED MAs as well. The assessment of the methodological quality of SCED MAs with a validated checklist is one way to determine how well-constructed SCED MAs are and offer insights into the confidence researchers can have in the validity of the results presented in these SCED MAs. So far, several reviews of SCED MAs have been conducted over the past few years (Beretvas \& Chung, 2008a; Farmer, Owens, Ferron, \& Allsopp, 2010; Maggin, O'Keeffe, \& Johnson, 2011; Schlosser et al., 2008; Shadish \& Rindskopf, 2007), but none of them has investigated the methodological quality of SCED MAs. Despite the importance of the quality of SCED MAs, there remains a paucity of evidence focused on reviewing this issue. In view of an expanding body of SCED MAs, we aimed to systematically investigate the methodological quality of SCED MAs. To the best of our knowledge, this review is the first study that systematically appraises the methodological quality of SCED MAs. The specific objective of our review is to provide an overview of the quality of SCED MAs published between 1985 and 2015, highlight the methodological shortcomings that can affect low quality evidence, and provide practical suggestions to improve the methodological quality of future SCED MAs.

\section{Methods}

\subsection{Search procedure}

SCED reviews (syntheses, SRs, and MAs) were searched. Among them, only studies that statistically integrated results of SCEDs were included in this review. In the current review, we refer to the eligible studies as SCED MAs. Reviews were identified by systematically searching electronic databases, previously published SCED reviews, relevant journals, bibliographies of relevant studies, citation indexes, and by contacting experts. This search was done by three people. In a first step, six electronic databases were searched: PsycINFO, Web of Science, Science Direct, Medline PubMed, ERIC, and CINAHL. Afterwards, a search for relevant metaanalyses in previously published reviews of SCED meta-analyses was conducted (Beretvas \& Chung, 2008; Farmer et al., 2010; Maggin, O'Keeffe et al., 2011; Schlosser et al., 2008; Shadish \& Rindskopf, 2007). To avoid publication bias, we searched grey literature (i.e., non-published literature) as well. To that end, ten databases were searched: the CORDIS Library, EdITLib, the Grey Literature Database of the Canadian Evaluation Society, the Index of Conference Proceedings, the Index to Theses in Great Britain and Ireland, IBSS, ProQuest Dissertations \& Theses, SSRN eLibrary, SIGLE, and Theses Canada. The search string used in all these databases consisted of the following combination of terms: ("single case" OR "single subject" OR "N of 1 " OR "small N" OR "multiple baseline design" OR "alternating treatments design" OR "reversal design" OR "withdrawal design") AND ("meta-analysis" OR "synthesis" OR "review"). There are different types of SCEDs and different synonyms such as multiple baseline design, alternating treatments design, reversal design, and withdrawal design. We defined these different types of SCEDs and synonyms as search terms to retrieve more eligible studies.

Next, we conducted a hand search of journals that showed up at least three times in the previous search steps: Advances in Learning and Behavioral Disabilities, American Journal of Mental Retardation, American Journal of Speech-Language Pathology, Augmentative and Alternative Communication, Behavioral Analyst Today, Behavior Modification, Behavior Therapy, Behavioral Disorders, Education and Treatment of Children, Evidence-Based Communication Assessment and Intervention, Exceptional Children, Journal of Autism and Developmental Disabilities, Journal of Behavioral Education, Journal of Clinical Epidemiology, Journal of School Psychology, Journal of Special Education, Learning Disability Quarterly, Neuropsychological Rehabilitation, Remedial and Special Education, Research in Autism Spectrum Disorders, Research in Developmental Disabilities, Review of Educational Research, and School Psychology Review.

Afterwards, the titles, abstracts and in some cases the full text of the retrieved studies were reviewed to identify studies meeting all inclusion criteria. In the next step, an ancestral search was conducted in the reference lists of eligible studies to identify additional relevant studies for inclusion in our review. After that, the Science Citation Index was consulted in order to see which studies cited the already retrieved studies in the previous search steps. Finally, the authors whose names appeared more than three times in the eligible studies were contacted and asked whether they had any other eligible studies.

\subsection{Inclusion/exclusion criteria}

We searched for published and unpublished studies between 1985 and 2015, written in English, which met the following four inclusion criteria:

(1) The study must include human participants;

(2) The study should be a quantitative review of at least two SCED studies (possibly in addition to group studies). Any kind of commentaries, reviews of qualitative case studies, reviews of group studies (including randomized controlled trials), conceptual and methodological studies without an empirical illustration were excluded;

(3) The study had to report a numerical summary of the treatment effect over all SCED studies. From the reviews of both SCED and group studies, only those that did separate analyses on the SCED data were included. This numerical summary of the treatment effect can be a summary of raw data (e.g., combined using multilevel models) and/or of effect size data, e.g., the standardized mean difference (SMD), regression coefficients, the Percentage of Non-overlapping Data (PND), the Percentage of All Nonoverlapping Data (PAND), or the Percentage of Data Points Exceeding the Median (PEM);

(4) The study had to be a journal article, conference paper, book, book chapter, dissertation, or report. Abstracts and slide presentations were excluded. 


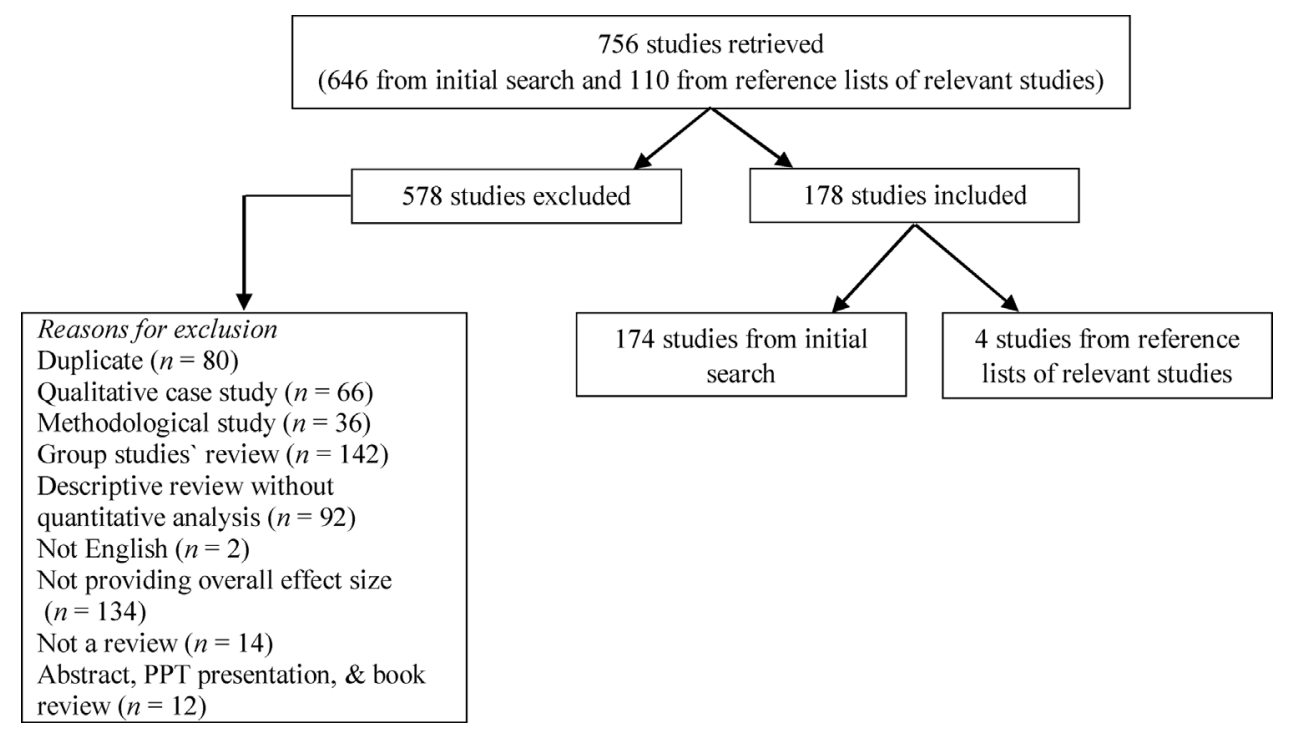

Fig. 1. Flowchart of the identification, inclusion, and exclusion of SCED MAs in the current review.

The list of all included and excluded studies is available upon request from the first author.

Fifty-one percent (384 out of 756 studies) of the retrieved studies were randomly selected and double-screened by two independent assessors judging whether the studies met all inclusion criteria; the remainder was screened by the first author only. Interrater agreement of screening the retrieved studies was calculated through dividing the number of agreements by the number of agreements plus the number of disagreements. In the case of disagreement, the assessors discussed the paper until they agreed on inclusion or exclusion. If no agreement was reached, the last author of this paper decided on inclusion or exclusion. Fig. 1 illustrates the number of included and excluded studies based on different reasons.

In total, the work was divided among five coders working in the field of MA of SCEDs, and all data from the included studies were extracted by them in the specific designed codebook. A detailed manual to guide the interpretation of R-AMSTAR items for this study was created to ensure consistency in the assessment. Twenty-two percent (40 out of 178 studies) of the included studies were randomly selected and coded independently by the second coder to assess the reliability of the coding system. Interrater agreement of data extraction was calculated in the same way as described in the previous paragraph. Disagreements were solved through discussion between coders. If no consensus was reached, the last author of this paper decided on the code given.

\subsection{Assessment of methodological quality}

Several methodological quality assessment tools applicable for SRs and MAs exist. Zeng et al. (2015) systematically reviewed 21 methodological quality assessment tools. Among these tools, Sack's Quality Assessment Checklist, Overview Quality Assessment Questionnaire (OQAQ), AMSTAR, checklists developed by Critical Appraisal Skills Programme (CASP, 2013), and National Institute for Clinical Excellence (NICE) are particularly used for assessing the quality of SRs and MAs. Beside these checklists, we reviewed the Scottish Intercollegiate Guidelines Network (SIGN, 2012) checklist, which is based on AMSTAR (Shea et al., 2007), Preferred Reporting Items for Systematic Reviews and Meta-Analyses (PRISMA; Moher et al., 2009) and R-AMSTAR (Kung et al., 2010) as well. For our study, we found AMSTAR appealing because it is more comprehensive compared to other tools, because it can be applied to SRs of non-randomized experiments as well, and because it is a widely accepted and common tool used by different associations and institutions. However, Burda, Holmer, and Norris (2016) and Faggion (2015) noted some limitations of AMSTAR in that some items and their instruction are not clear enough and need to be revised. Whereas it is possible that some SRs partially meet the items' criteria, there is no possibility to differentiate between studies that meet all criteria or partially meet the criteria. Although AMSTAR (Shea et al., 2007) has good agreement, reliability, construct validity, and feasibility to evaluate the methodological quality of SRs (Popovich et al., 2012), it fails to produce quantifiable assessments of methodological quality of SRs. Therefore, Kung et al. (2010) proposed a revised checklist, the R-AMSTAR checklist, that is meant to keep the content and construct validity, and to utilize the criteria originally from AMSTAR, and moreover is more successful in quantifying the quality of SRs compared to AMSTAR because it assesses more criteria separately. This tool, as AMSTAR, has 11 main individual items, with detailed criteria to quantify these 11 domains (Kung et al., 2010). The larger the sum of the score for each item, the higher the methodological quality of the SRs/MAs. The validity of R-AMSTAR has been investigated and supported in studies by Kung et al. (2010) and Shea et al. (2009).

We decided to use the R-AMSTAR (like other researchers, such as Alarcón et al., 2017; Bao et al., 2014; Brossart et al., 2008; Corry et al., 2015; Deckert et al., 2014; Faggion \& Giannakopoulos, 2013; Kowalczuk et al., 2015; O’Donnell et al., 2014; Parke et al., 2015; Pearce et al., 2015; Pinnock et al., 2017; Popovich et al., 2012; Ramchandani et al., 2010; Rotta et al., 2015; Schmitter, Sterzenbach, Faggion, \& Krastl, 2013; Sygouros \& Acar, 2013; Thompson, Schabrun, Romero, Bialocerkowski, \& Marshall, 2016; Wei et al., 2015; 
Wells et al., 2013) because of its better and clearer guidance for quality assessment process and because of its higher transparency. Yet, as Pieper et al. (2014) noted, there are still some issues with specific items. In addition, the formulation of some items does not fit well for SCED MAs. Therefore, we modified some criteria of the original R-AMSTAR. The modified R-AMSTAR constitutes 11 main items and in total 44 criteria (see Table 2 for all details related to the main items and their criteria; the modified criteria are indicated with an asterisk). A first modification is related to the second item of the original R-AMSTAR that focuses on data extraction but ignores study selection (Bao et al., 2014). Therefore, we added three more criteria related to study selection (2A, 2B, and 2C) to this item. Next, we separated one of the criteria of the third item of the original R-AMSTAR; 'the report must include years and databases used (e.g. Central, EMBASE, and MEDLINE)', into two criteria ('3B: publishing time range of the included studies are mentioned' and '3C: used databases are mentioned'). We did this because the multidimensionality of the item hampers the scoring. We broke one of the criteria of Item 7; 'quality of evidence should be rated/ranked based on characterized instruments', into two criteria ('7D: quality of evidence is rated/ranked over all included studies based on characterized instruments ' and '7E: quality of evidence is rated/ranked for each individual study based on characterized instruments'), because the information about the scientific quality of primary studies in an overall form or individually for each primary study cannot be considered to be the same facet of quality. Two criteria of Item 8; 'to have conclusions integrated/drives towards a clinical consensus statement' and 'this clinical consensus statement drives toward revision or confirmation of clinical practice guidelines', were modified to provide just one criterion ('8C: conclusions integrated/ derived towards practice guidelines and recommendations'). One criterion of Item 9 was omitted because we found it less relevant for SCED MAs; 'if homogeneity exists, the author should state a rationale or a statistical test'.

In assessing the methodological quality of the included studies, we (like Rotta et al., 2015; Rave \& Rumohr, 2016) preferred not to use the scoring system of the original R-AMSTAR, in which each item score ranged between 1-4 and the total quality score ranged from 11 to 44 (Kung et al., 2010). In this way of scoring, even if the study does not meet any criterion of the specific item, it would be assigned a 1 for each item and the study would obtain a final total score of 11 . We therefore calculated the methodological quality score in two alternative ways. In the first method, we allocated 1 to each criterion if the study explicitly met the criterion, otherwise the score assigned was 0 (referring to 'no', 'not reported' or 'not applicable'). Then, the total quality score was calculated by summing all the scores of all the criteria met. Based on this way of scoring, the total score ranged from 0 to 44 . In the second method, for each of the 11 items, the scores were calculated by dividing the number of criteria satisfied within each specific item by the total number of criteria for that item. Therefore, under this method of scoring, the score for each item ranged from 0 to 1 . The total quality score, defined as the sum of the scores of the 11 items, ranged from 0 to 11 . Through this second method of scoring, it was also possible to control for the different numbers of criteria that each item had (e.g., one item had three criteria and another item had six criteria). For both scoring methods, a higher quality score indicates a higher methodological quality, higher internal validity, and lower risk of bias.

\section{Results}

The initial electronic search (i.e., the search of electronic databases and grey literature, and the hand search of the journals) resulted in 646 papers. After removing the duplicates, a final screening of the abstracts, titles, or whole text of retrieved studies resulted in 174 eligible studies that met our inclusion criteria. Searching the bibliographical lists of these eligible studies identified 110 additional studies from which only four studies met the inclusion criteria. In total, we therefore retrieved 756 studies, but only 178 studies met all inclusion criteria for the present review. The interrater agreement for screening and identifying the eligible studies was $87 \%$. All disagreements were resolved through discussion until consensus was reached for all included and excluded studies. Based on these discussions, inclusion/exclusion criteria were further clarified and the eligibility of all studies was assessed again. Two of the included studies reanalyzed an existing data set (as an illustration of specific methodologic proposals). In those cases, the information was extracted from the original source rather than from the included studies themselves. The average interrater agreement for data extraction was $95 \%$.

Most of these SCED MAs (93\%) were published in 58 different journals and the rest of SCED MAs (7\%) were dissertations, book chapters, and project reports. Almost one third (32\%) of journal articles were published in Research in Developmental Disabilities (12 studies), Remedial and Special Education (11 studies), School Psychology Review (10 studies), Exceptional Children (10 studies), and Journal of Behavioral Education (10 studies). SCED MAs focused on a variety of populations and also interventions. The most frequent interventions of interest among them were behavioral interventions ( $22 \%$ of studies). Other kinds of interventions such as video modeling interventions, augmentative and alternative communication (AAC) interventions, academic interventions, reading interventions were also observed. SCED MAs reviewed in current study varied in the population of interest, but the main population consisted of people with autism spectrum disorder (ASD, $24 \%$ of studies). Other frequently mentioned groups of interest were people with behavioral disorders, developmental disabilities, learning disabilities (LD), or other kinds of disabilities.

As the results showed, studies used various kinds of effect sizes to express the effect observed in primary SCEDs (applying different metrics to calculate the primary effect sizes such as SMD, PND, PAND, PEM, ...) and synthesized these effect sizes by providing the mean, median, or range or by doing regression or multilevel analyses; other studies performed multilevel meta-analyses for aggregating the SCED raw data. Multiple analyses were done in some SCED MAs. Most of the MAs synthesized results by calculating the mean, median or range of the individual effect sizes from primary SCED studies (90\% of studies). Small proportion of SCED MAs aggregated the results by doing regression analysis of individual effect sizes from primary studies (4\% of studies). Some other MAs applied multilevel analysis to obtain the overall treatment effect (22\% of studies).

The last column of Table 1 gives for each item the mean and median score, and its correlation with the total quality score (obtained through the second way of scoring explained in the previous paragraph). The values of means and medians ranged from 0 


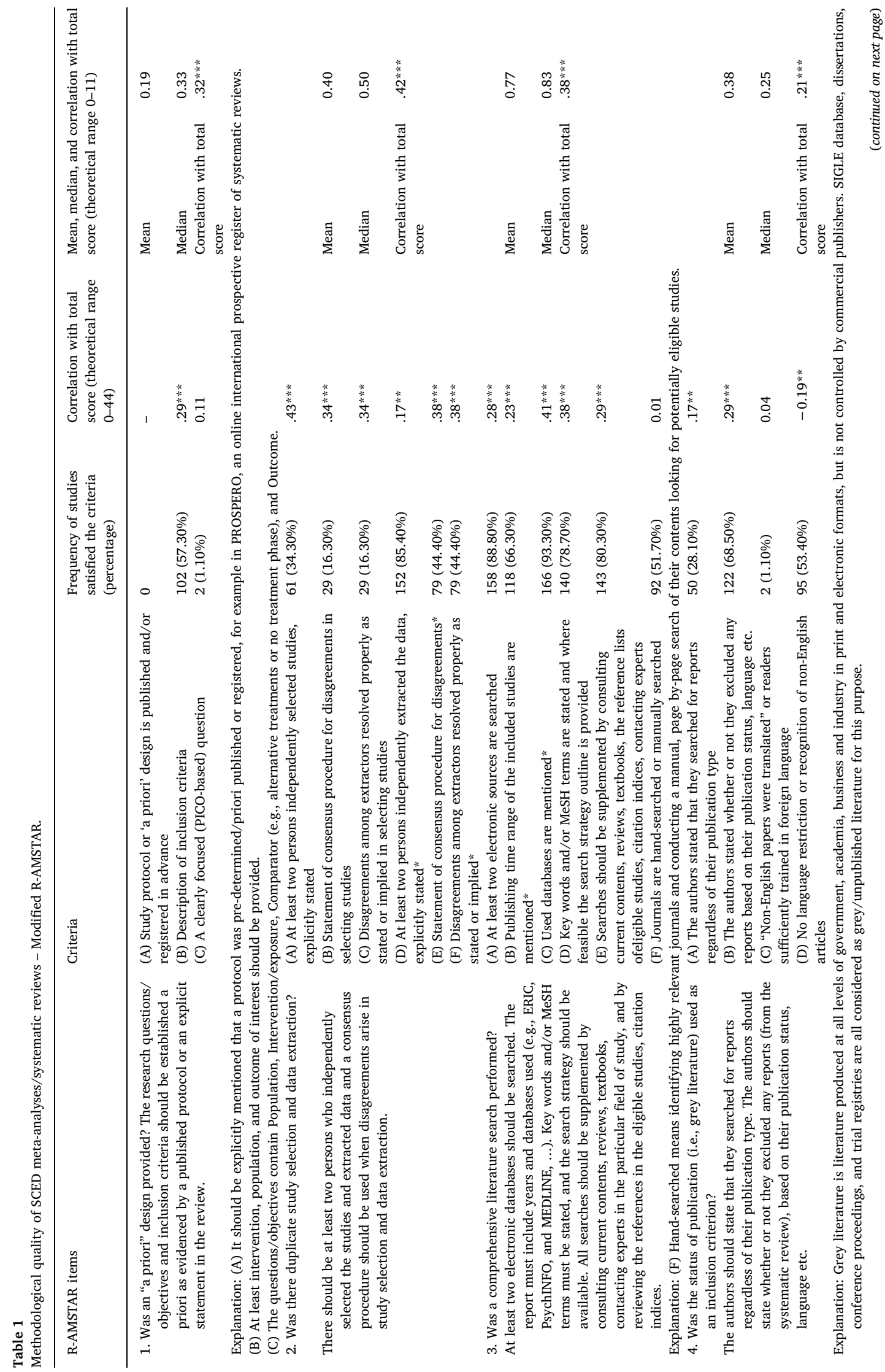




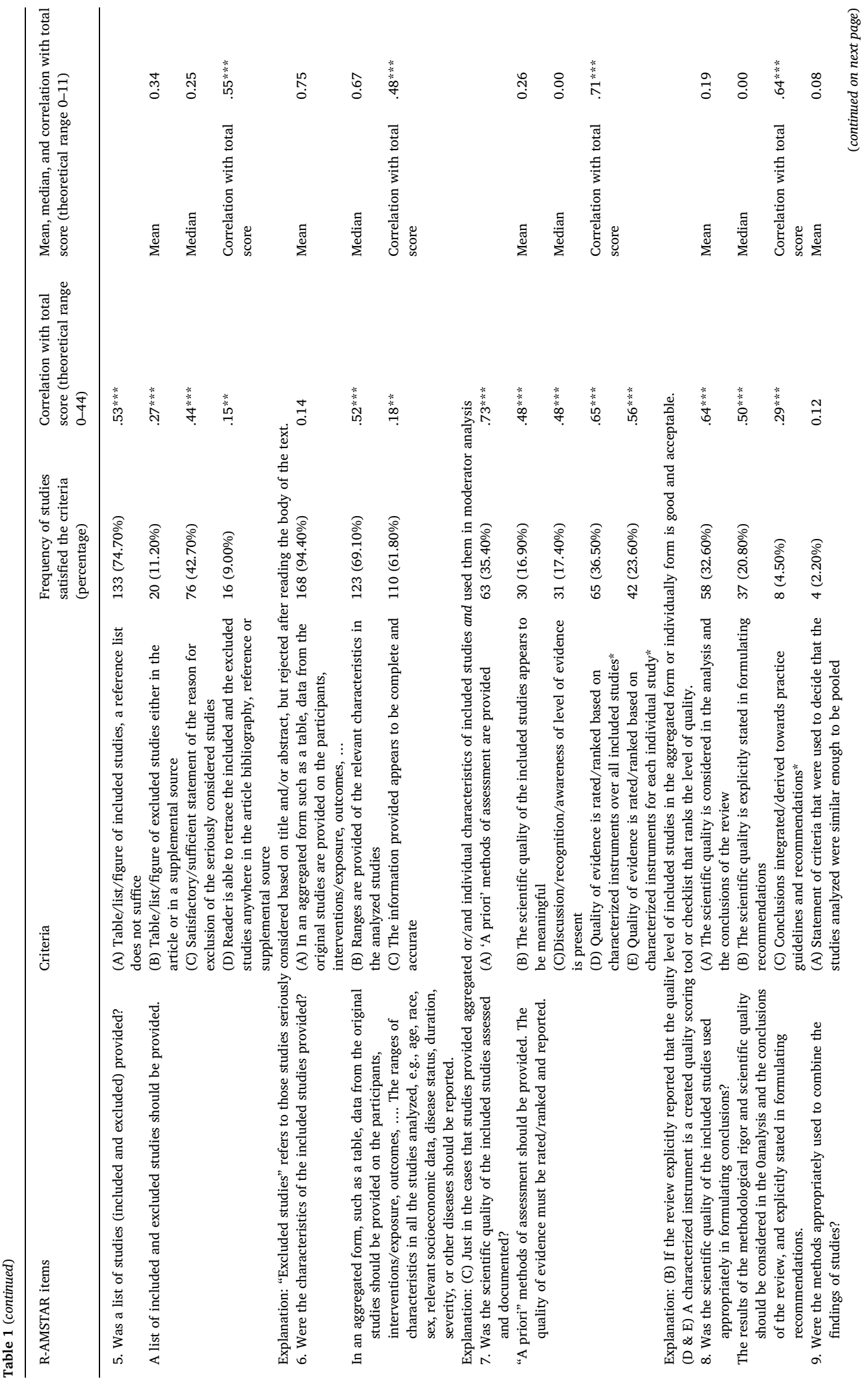




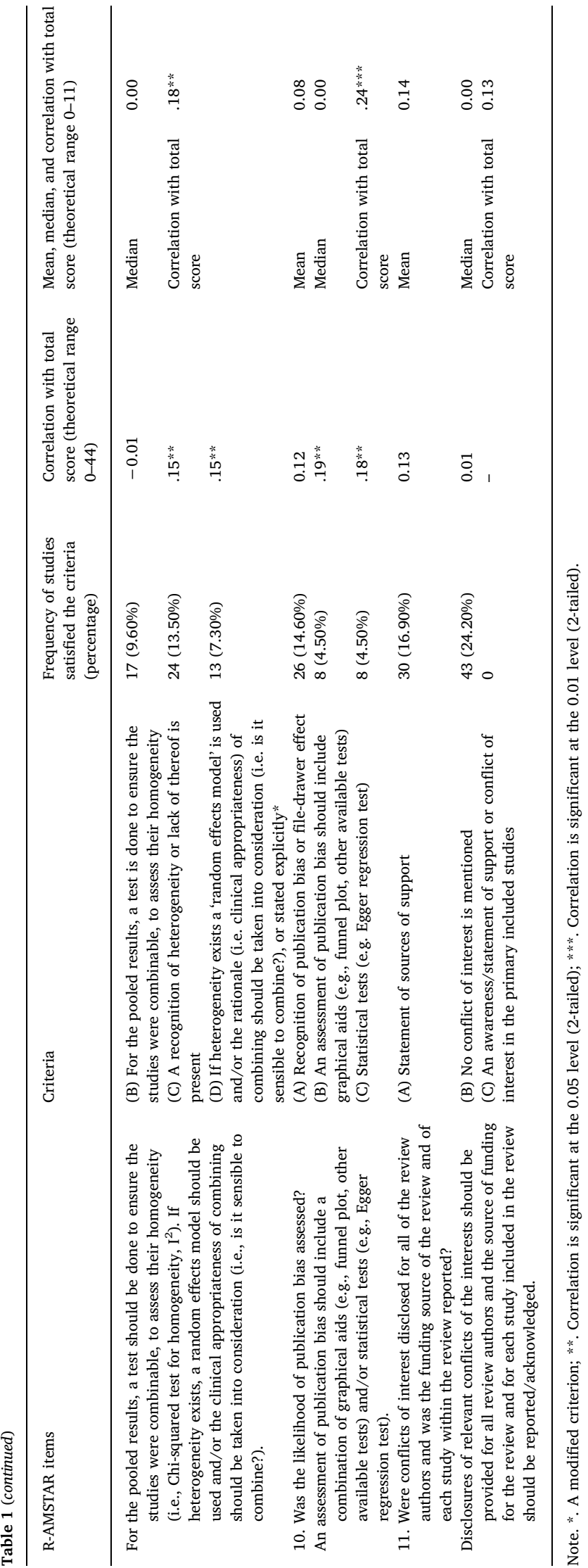


Table 2

Inclusion criteria in included studies.

\begin{tabular}{llc}
\hline Inclusion Criteria & Frequency & Percentage \\
\hline The time range of publishing & 115 & $64.60 \%$ \\
The population of interest & 157 & $88.20 \%$ \\
The intervention(s) of interest & 152 & $85.40 \%$ \\
The outcome(s) of interest & 134 & $75.30 \%$ \\
The type of study designs & 141 & $79.20 \%$ \\
The kind of data & 104 & $58.40 \%$ \\
The geographical location or setting & 32 & $18.00 \%$ \\
The language & 82 & $46.00 \%$ \\
The publication type & 90 & $51.00 \%$ \\
The study quality & 5 & $2.80 \%$ \\
\hline
\end{tabular}

to 1 , because scores for each item are calculated based on the number of satisfied criteria of the item divided by the total number of all criteria of that item. The two other columns show the frequencies and percentages of the studies that satisfied the specific criteria of each item, and the correlations of the criteria with the total quality score (this time obtained through the first way of scoring explained earlier). More explanations about the items and criteria are provided as well.

\subsection{Item 1: was an "a priori" design provided?}

As is evident from the results in Table 1, only a small proportion of the studies provided the 'a priori' design of the SCED MA. None of the MAs explicitly mentioned that they published the meta-analytic study's design in advance. Around half of the studies provided at least the intervention, the population, and the outcome of interest as part of their inclusion criteria. As shown in Table 2, the majority of the studies mentioned the population of interest, intervention of interest, type of study design, and outcome of interest, respectively.

As can be seen in Table 3, the comparison of interest was only provided in three of the SCED MAs that were reviewed. Only two studies developed their research questions in a complete PICO format (including information on Population, Intervention, Comparison, and Outcome). The rest of the studies did not develop the question related to the comparison of interest (i.e., studies did not mention if the comparison of interest was between treatment and no-treatment conditions or between treatment and alternative treatments).

\subsection{Item 2: was there duplicate study selection and data extraction?}

Related to performing duplicate study selection and data extraction, the mean and median quality score across all SCED MAs were 0.4 and 0.5 respectively. In $61(34.30 \%)$ studies it was stated that at least two people independently selected eligible studies and in a total of $29(16.30 \%)$ studies, the procedure for solving disagreements through a final screening was described. From these studies, consensus was reached in two studies with disagreements properly resolved through discussion. In eight studies, a third rater made an evaluation in the case of discrepancy and made the final decision to resolve the disagreement. In 152 (85.40\%) of the SCED MAs, at least two people independently extracted data and 79 studies (44.40\%) provided information about how disagreements were resolved. Out of those SCED MAs, it was reported in 74 studies that disagreements related to data extraction were resolved through discussion and more clarification. In five studies, a third rater evaluated and made decisions in the case of discrepancies.

\subsection{Item 3: was a comprehensive literature search performed?}

The proportion of SCED MAs that conducted a comprehensive literature search was higher compared to the proportion obtained in other items. In total, $88.80 \%$ of the SCED MAs searched in at least two electronic databases and 143 studies reported supplementing the searches through checks of the reference lists of eligible studies, citation indexes, or contacting experts. MEDLINE was the most frequently searched electronic database. In 92 studies, the journals were manually searched. What stands out in Table 1 is that a large percentage of the studies $(93.30 \%)$ mentioned the use of electronic databases. Different search sources are presented in more detail in Table 4 . From the figures in Table 4, it is apparent that electronic databases and reference lists are the most common search sources.

Table 3

The PICO format of research questions of included studies.

\begin{tabular}{lll}
\hline Provided information & Frequency & Percentage \\
\hline Population of interest & 128 & $71.90 \%$ \\
Intervention(s) of interest & 154 & $86.50 \%$ \\
Comparison of interest & 3 & $1.70 \%$ \\
Outcome(s) of interest & 120 & $67.40 \%$ \\
\hline
\end{tabular}


Table 4

Search strategies of included studies.

\begin{tabular}{lll}
\hline search strategies & Frequency & Percentage \\
\hline Electronic databases (using queries) & 171 & $96.10 \%$ \\
Grey literature databases & 39 & $21.90 \%$ \\
Hand searching journals & 92 & $51.70 \%$ \\
Searching reference lists (backward searches) & 138 & $77.50 \%$ \\
Using citation indices (forward searches) & 10 & $5.60 \%$ \\
Contacting experts and/or authors & 21 & $11.80 \%$ \\
Primary studies of previous similar reviews & 7 & $3.90 \%$ \\
Not reported & 2 & $1.10 \%$ \\
\hline
\end{tabular}

\subsection{Item 4: was the status of publication (i.e., grey literature) used as an inclusion criterion?}

The mean and median quality score of the item referring to the use of the status of publication (i.e., grey literature) as an inclusion criterion across all SCED MAs were 0.38 and 0.25 respectively. What is apparent from Table 1 is that just two studies included and translated non-English papers. Tables 1 and 4 show that 50 studies searched for studies regardless of their publication type and 39 studies explicitly reported that they searched grey literature. From Table 1, it can be seen that more than two-thirds (68.50\%) of the studies stated whether they excluded any reports based on their publication status, language, or study quality.

\subsection{Item 5: was a list of studies (included and excluded) provided?}

Concerning whether a list of studies (included and excluded) was provided, some SCED MAs provided the relevant information. The included studies in SRs/MAs was provided in the table, list, or figure in the text or supplemental source in 133 studies (74.70\%). Closer inspection revealed that typically primary studies were marked with an asterisk in the reference list (in $88.80 \%$ of the studies). Ten studies explicitly mentioned that the list of included studies could be requested by contacting one of the authors of that SR/MA. A small proportion of the studies (11.20\%) provided the excluded studies either in the text or supplemental source. Seven studies explicitly mentioned that the list of excluded studies could be obtained by contacting one of the authors of that SCED MA.

\subsection{Item 6: were the characteristics of the included studies provided?}

The characteristics of the primary studies were provided in many SCED MAs. In Table 1 we can see that a considerable number of studies (94.40\%) provided the retrieved data related to the participants, interventions, outcomes, and so on from the primary studies in aggregated form such as in a table. In $69.10 \%$ of the studies, the range of the relevant characteristics in the individual primary studies were presented. In $61.80 \%$ of the studies, the information provided appears to be complete and accurate, because the MAs performed moderator analysis using this information.

\subsection{Item 7: was the scientific quality of the included studies assessed and documented?}

A total of 74 studies (41.60\%) appraised and documented the scientific quality of primary studies. From these SCED MAs, 63 (35.40\%) studies provided 'a priori' methods for quality assessment of primary studies as shown in Table 1. The SCED MAs mostly applied the Horner et al. (2005) tool and What Works Clearinghouse (WWC; Kratochwill et al., 2010) standards for quality appraisal of SCED primary studies. Based on the information explicitly presented in the studies, the scientific quality of the primary studies appeared to be satisfactory in $16.90 \%$ of the studies. The discussion/recognition/awareness of the level of evidence was presented in only a small proportion of the studies (17.40\%). The quality of evidence was rated based on characterized instruments over all included studies more than one-third of the studies (36.50\%) and for each individual study in less than one-third of the studies (23.60\%).

\subsection{Item 8: was the scientific quality of the included studies used appropriately in formulating conclusions?}

The mean and median quality score related to using the scientific quality of the included studies appropriately in formulating conclusions were 0.19 and 0.00 respectively across all SCED MAs. Almost one-third of the studies (32.60\%) considered the scientific quality of primary studies in the analyses and the conclusions of the reviews. Approximately one-fifth (20.80\%) of the studies explicitly stated the scientific quality in formulating the recommendations. However, only in a few (4.50\%) studies were conclusions derived offering practice guidelines and recommendations.

\subsection{Item 9: were the methods appropriately used to combine the findings of studies?}

Only a small minority of the studies used appropriate methods or reported an assessment of the appropriateness of combining the studies in a quantitative way. The results reveal that $13.50 \%$ of the SCED MAs presented a recognition of the existence of 
heterogeneity of pooled studies or a lack thereof. Few studies stated the criteria to decide whether the included studies were similar enough to be pooled (2.20\%). Some studies performed a statistical test to assess the homogeneity of the primary studies (9.60\%). If heterogeneity existed a 'random effects model' was used and/or the rationale (i.e. clinical appropriateness) of combining was taken into consideration (i.e. is it sensible to combine?), or stated explicitly in a small proportion of the studies (7.30\%).

\subsection{Item 10: was the likelihood of publication bias assessed?}

A majority of the SCED MAs did not report an assessment of the likelihood of publication bias. Some studies (14.60\%) recognized the possibility of a publication bias or file-drawer effect, but only a few studies (4.50\%) used statistical tests such as Egger's regression test, or included graphical aids such as funnel plot (4.50\%) to assess potential publication bias.

\subsection{Item 11: were conflicts of interest disclosed for all of the review authors and was the funding source of the review and of each study within the review reported?}

A small minority of the SCED MAs disclosed the conflicts of interest for all of the review authors and reported the funding source of the review and of each study within the review. Across all studies, $24.20 \%$ mentioned no conflict of interest and $16.9 \%$ studies stated the sources of financial support. What stands out in Table 1 is that none of the SCED MAs gave information about the support or the conflict of interest in the primary included studies.

\subsection{The total methodological quality}

The mean and median of the total methodological quality score of the 178 SCED MAs based on the first method of scoring were 15.57 and 16 respectively (range: 4-27, interquartile range[IQR]: $12-19, \mathrm{SD}=4.80$ ). Remember that this score is the sum of all criteria that were satisfied using the modified R-AMSTAR and has a theoretical range from 0 to 44 . Closer inspection of the quality scores distribution indicates that $93.80 \%$ of the total scores are less than 22 , which is the midpoint of the possible range of scores. Fig. 2 shows the methodological quality distribution of the included studies calculated based on the first method of scoring.

The mean and median of methodological quality scores of 178 SCED MAs based on the second method of scoring were 3.58 and 3.59 respectively (range: $1.08-6.22$, interquartile range[IQR]: $2.75-3.59$, SD $=1.13$ ). These scores are the sum of the scores of all 11 items of the modified R-AMSTAR, and therefore could in theory range from 0 to 11 . Almost all (97.60\%) quality scores are equal or less than 5.5, the middle point of the range of possible scores. Fig. 3 shows the methodological quality distribution of the included studies calculated based on the second method of scoring.

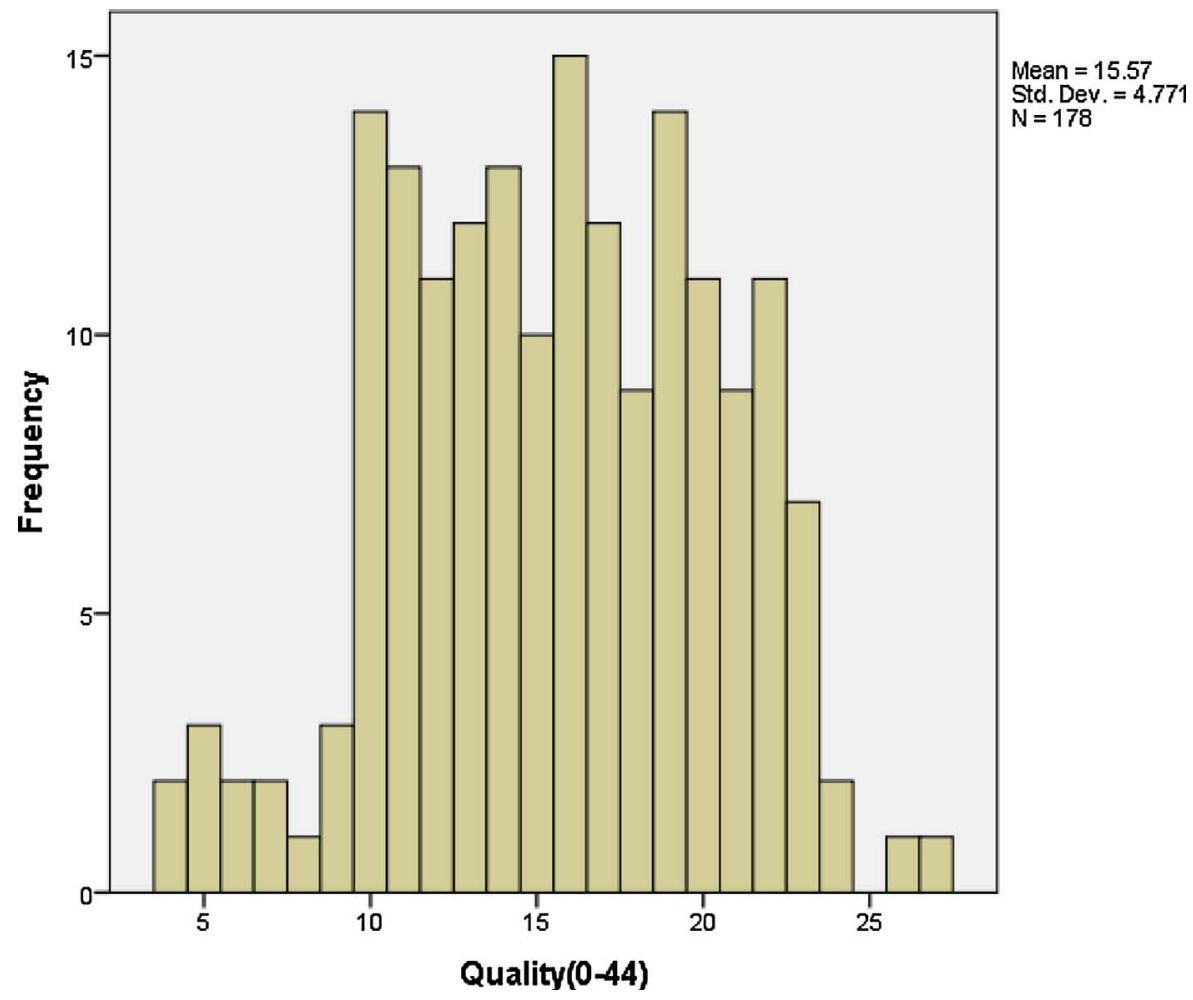

Fig. 2. Methodological quality distribution (0-44) of the SCED MAs. 


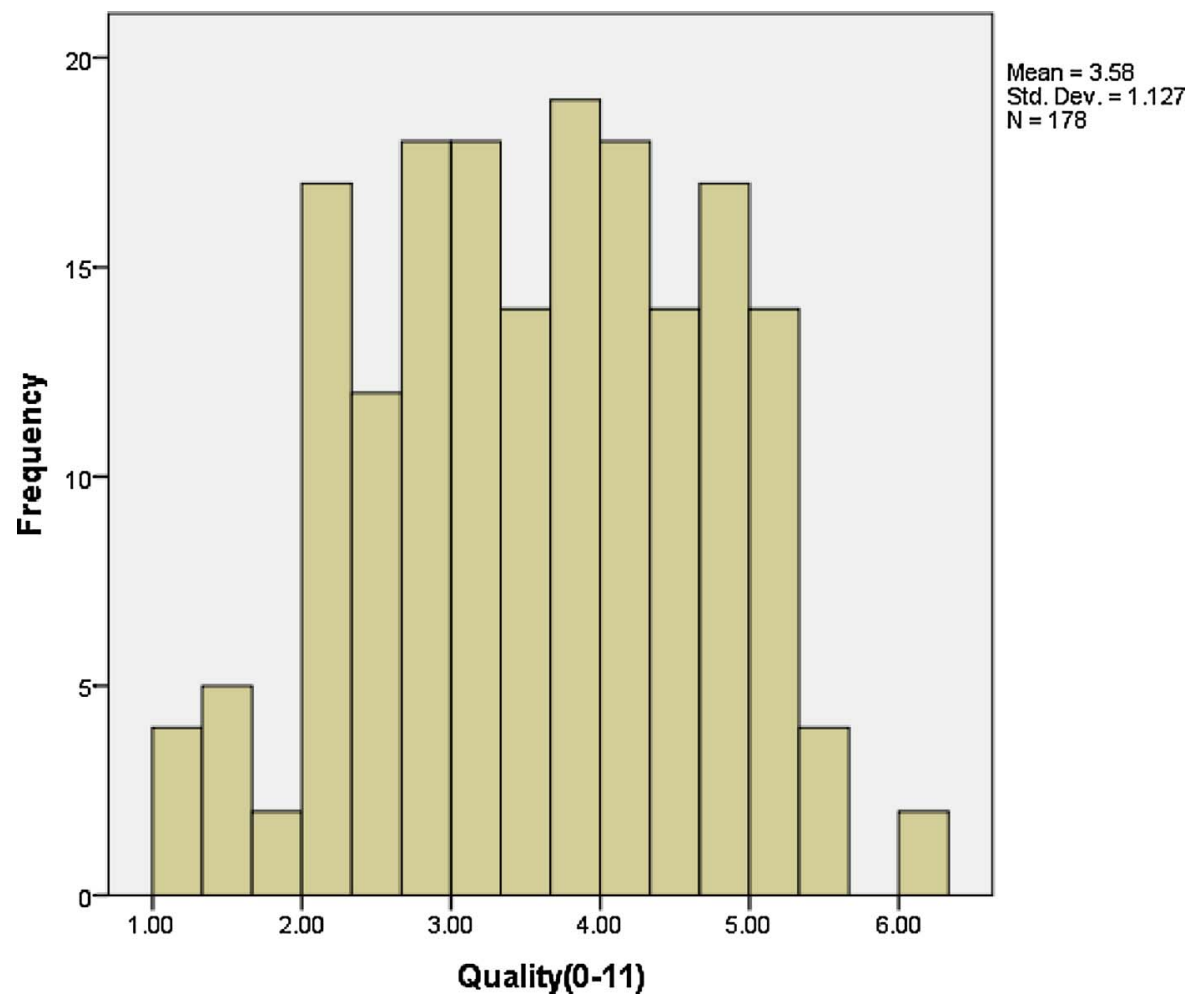

Fig. 3. Methodological quality distribution (0-11) of SCED MAs.

A one-way ANOVA was used to check whether there are any significant differences between journals in the methodological quality of the studies. In this analysis, we only included journals in which 10 or more studies were published (Research in Developmental Disabilities, Remedial and Special Education, School Psychology Review, Exceptional Children, and Journal of Behavioral Education). No significant differences between journals were found $\left(F(4,48)=1.67, p=.17, \eta_{p}{ }^{2}=.12\right)$.

A regression analysis was performed to test whether there was evidence of a time trend in the methodological quality of SCED MAs. The results of the regression analysis indicated a significant positive effect of year of publication on methodological quality over the last three decades $(\beta=0.36, p<.01, t(176)=5.11)$ and time can explain $13 \%$ of the variance in methodological quality $\left(R^{2}=.13, F(1176)=26.15, p<.01\right)$. Fig. 4 visualizes the trend over time, using a linear equation, and using two different smoothing methods: using a cubic spline and using locally weighted scatterplot smoothing (LOESS). As can be seen, the smoothing spline actually does not differ much from the linear fit. The LOESS curve suggests that next to the increasing trend over the years (which could already be observed from the linear fit), a slight decline in the most recent years (starting from 2012 to 2013) can be observed. We also used Tufte-style sparklines to study time trends for each methodological quality item, but they did not show clear patterns.

To study further the evaluation of quality over time, primary studies were divided into four groups according to the year of publication: papers published between 1985 and 2000 were grouped together (because there were not many articles published in this period), studies published between 2001 and 2005, between 2006 and 2010, and between 2011 and 2015 were grouped into a second, third and fourth category, respectively. As shown in Table 5, the mean and median of the methodological quality of the SCED MAs increased from 1985 to 2015. Upon closer inspection, we found that this increase of the methodological quality over time can be primarily attributed to the increase in some items such as 'was an “'a priori”' design provided?' (Item 1), 'was there duplicate study selection and data extraction?' (Item 2), 'was the scientific quality of the included studies assessed and documented?' (Item 7), and 'was the scientific quality of the included studies used appropriately in formulating conclusions?' (Item 8).

\section{Discussion}

MAs and SRs are sometimes considered the highest methodological level in research studies and their findings usually lead to formulations of clinical guidelines and guide decision-making processes. However, not all published SRs and MAs have the same methodological rigor and validity. Despite the increasing interest in using SCEDs to evaluate the effectiveness of a treatment and the large amount of methodological work that has been done in the last two decades, there still is no consensus on appropriate and standard methods for synthesizing the results of these studies (Beretvas \& Chung, 2008a; Shadish, Hedges, Horner, \& Odom, 2015; Shadish \& Rindskopf, 2007). In the current review, we focused on SCED MAs with numerical summaries of the overall treatment effect, regardless the methods applied for obtaining this overall effect size. We used a modified R-AMSTAR, including 44 criteria 


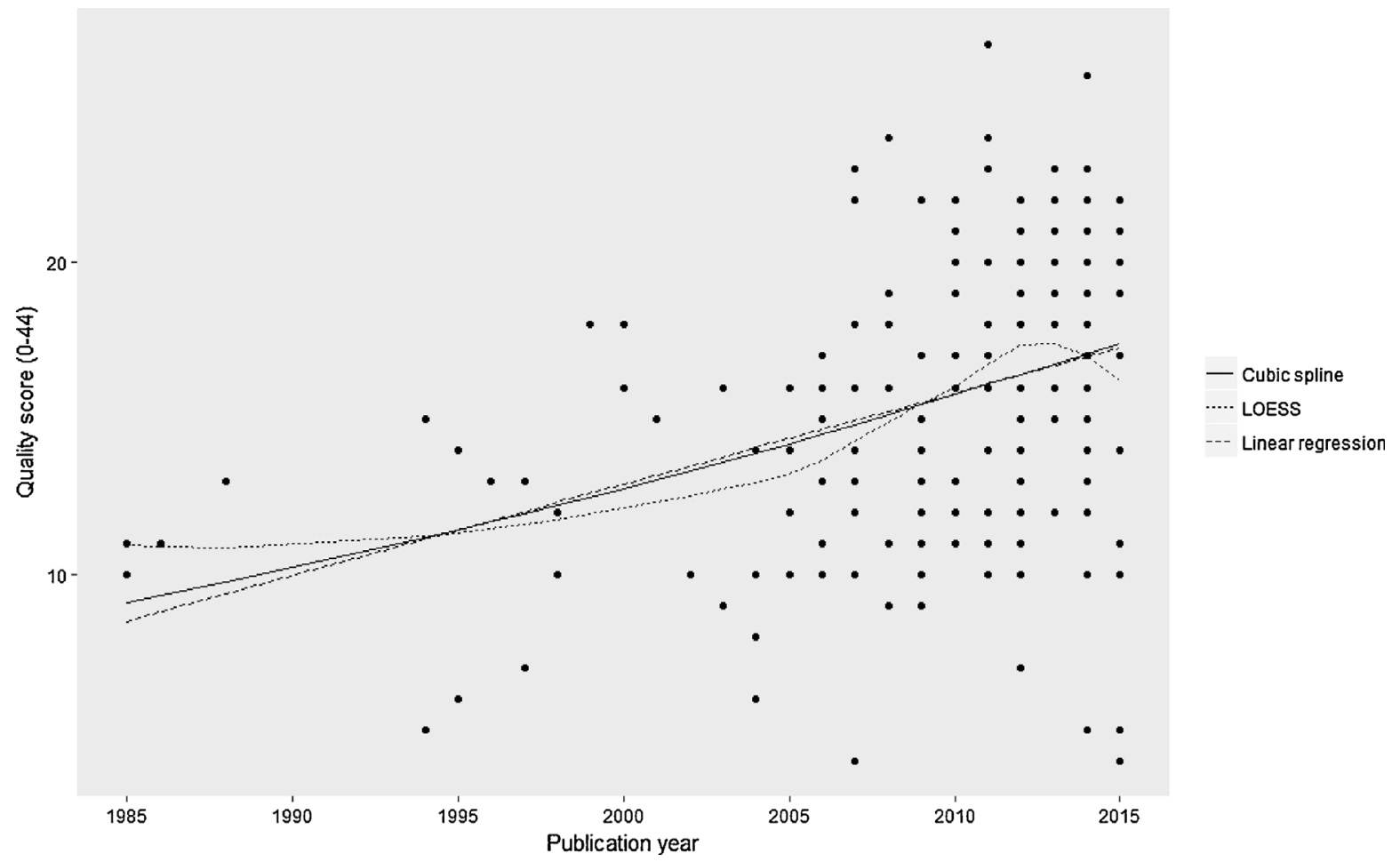

Fig. 4. Methodological quality over time.

Table 5

The evolution of methodological quality of the SCED MAs.

\begin{tabular}{|c|c|c|c|c|c|c|c|c|c|}
\hline \multirow[t]{2}{*}{ Time range } & \multirow[t]{2}{*}{ Total number of studies } & \multicolumn{4}{|c|}{ Quality score (0-44) } & \multicolumn{4}{|c|}{ Quality score (0-11) } \\
\hline & & Mean & Median & Range & Standard deviation & Mean & Median & Range & Standard deviation \\
\hline 1985-2000 & 16 & 12.00 & 12.50 & $5.00-18.00$ & 3.86 & 2.74 & 2.79 & $1.08-4.17$ & 0.93 \\
\hline 2001-2005 & 12 & 11.67 & 11.00 & $6.00-16.00$ & 3.31 & 2.68 & 2.63 & $1.42-3.92$ & 0.81 \\
\hline 2006-2010 & 56 & 14.93 & 14.50 & $4.00-24.00$ & 4.36 & 3.40 & 3.33 & $1.08-5.32$ & 1.00 \\
\hline 2011-2015 & 94 & 17.06 & 18.00 & $4.00-27.00$ & 4.66 & 3.95 & 4.14 & $1.08-6.22$ & 1.10 \\
\hline Total & 178 & 15.57 & 16.00 & $4.00-27.00$ & 4.77 & 3.58 & 3.59 & $1.08-6.22$ & 1.13 \\
\hline
\end{tabular}

grouped in 11 items, to obtain an idea of the methodological quality of 178 SCED MAs, by examining the distribution of both the total score of the methodological quality of SCED MAs, as well as the score on individual criteria.

The findings reveal low methodological quality among SCED MAs. According to Table 5, most reviews did not achieve even the middle R-AMSTAR score, which is an indication that the quality of the reviews could be improved. Our finding is similar to the finding of Schmitter et al. (2013) who did not focus on SCED studies, but also found a low mean quality score, indicating that most of the studies did not reach a high quality level.

\subsection{The methodological quality items with the highest scores among SCED MAs}

While the overall methodological quality of SCED MAs was somewhat disappointing, there were also items for which the quality scores were good. The items that reached the highest quality scores are 'was a comprehensive literature search performed?' (Item 3), which is similar to the findings of other reviews (Alarcón et al., 2017; Bao et al., 2014; Deckert et al., 2014; Faggion \& Giannakopoulos, 2013; Sygouros \& Acar, 2013), and 'were the characteristics of the included studies provided?' (Item 6), which also reached a high score in the reviews of Alarcón et al. (2017), Bao et al. (2014), Faggion and Giannakopoulos (2013), Kowalczuk et al. (2015), Schmitter et al. (2013), and Sygouros and Acar (2013). As the numbers in Table 1 indicate, a considerable percentage of the studies have performed a comprehensive literature search in different electronic databases and supplemented their search with hand searching the relevant journals or other possible sources such as reference list of eligible studies, citation indexes, or contacting the experts (Item 3). In a well-conducted SR or MA, the search strategy to identify the relevant eligible studies should be clearly specified (Egger \& Smith, 2001). Conducting the systematic and comprehensive search in literature, selecting the available evidence based on the predefined inclusion/exclusion criteria, and analyzing the data according to the carefully determined question(s) can lead to well- 
conducted SRs (Shea et al., 2007). SRs that apply a rigorous methodology with clearly developed research questions and a comprehensive search strategy would be able to provide reproducible results and include all potentially eligible studies and reduce the bias and random errors (Remschmidt et al., 2014). Item 6 also obtained a higher score compared to other items; most of the studies presented the relevant characteristics of the primary studies in aggregated form or individually for all primary studies clearly and accurately and over two thirds of the studies used these characteristics properly in their analyses such as moderator analysis. Providing characteristics of primary studies can provide more insight to readers about the included studies and can be used by the systematic reviewers and meta-analysts for further analyses to explain more variability in the effect sizes attributed to these characteristics.

\subsection{The methodological quality items with the lowest scores among SCED MAs}

SCED MAs obtained the lowest quality scores in items such as 'were the methods appropriately used to combine the findings of studies?' (Item 9) and 'was the likelihood of publication bias assessed?' (Item 10). Regarding Item 9, only a few studies tested the heterogeneity. Alarcón et al. (2017), Bao et al. (2014), Deckert et al. (2014), Schmitter et al. (2013), and Wei et al. (2015) found similar results in their assessment of methodological quality of SRs/MAs. Checking heterogeneity across primary studies in MAs is important because aggregating heterogeneous studies can produce inaccurate and misleading treatment effects. Applying a random effects model can be a solution to account for heterogeneity and improve the accuracy of results, although it cannot explain or remove the primary study differences (Wells et al., 2013). However, our findings reveal that less than one-tenth of the studies applied a random effects model in case of heterogeneity. It should be considered that the greater the heterogeneity across primary studies, the more likely the lack of a precise overall effect size (Faggion \& Giannakopoulos, 2013) and therefore, not checking heterogeneity and not taking it into account can increase the error and bias in the results.

Item 10 was another problematic area, the assessment of the likelihood of publication bias by applying statistical test such as Egger's regression test or graphical aids such as funnel plot. This result is similar to the findings of some reviews in which the methodological quality of SRs and MAs was assessed (Alarcón et al., 2017; Bao et al., 2014; Deckert et al., 2014; Faggion \& Giannakopoulos, 2013; Kowalczuk et al., 2015; Popovich et al., 2012; Schmitter et al., 2013; Sygouros \& Acar, 2013; Wei et al., 2015). Testing publication bias is important, because studies with statistically significant results may be more likely to be published than non-significant studies. In the case of publication bias, the results of MAs might be impacted and cause overestimation of the intervention effect (De Vito et al., 2007). Analogously, in SCED research, studies without visible effects are less likely to be published resulting in the same sorts of potential publication bias". Since undetected publication bias may lead to imprecise or misleading results of SRs, statistical approaches such as the use of funnel plots and regression test proposed by Egger and colleagues have been developed and should be used to detect publication bias (Remschmidt et al., 2014). Consequently, the researchers should assess publication bias, by applying a graphical aid such as funnel plot and/or a statistical test such as the Egger regression test (Hall et al., 2017). Part of the explanation for why only a small number of SCED MAs assessed publication bias may be that assessing publication bias in SCED MAs is not as common as in group comparison design MAs. As Shadish et al. (2015) pointed out in their report, it may initially seem that publication bias is unlikely in SCED research because relatively few SCED researchers use statistics and the studies cannot be rejected for publication without statistical significance. Their experience suggests that publication bias may be present in the sense that visually detectable effects might have a higher probability of being published, since SCED researchers may decide to not publish the studies with visually insignificant effect even without calculating effect size. Our experience also suggests that visually detectable effects are more likely to be published, and hence it might be more prudent to use a method for evaluating the existence of publication biases like some of the included SCED MAs have done.

Generally, none of the included SCED MAs explicitly mentioned that they published or registered a study protocol or 'a priori' design and the majority of them did not construct their research questions/objectives based in the PICO (population, intervention, comparison, and outcome) format (Item 1). Developing a review protocol in advance is a good measure to avoid bias. An 'a priori' detailed study protocol can clearly address the review questions, the subgroups of interest, the methods and inclusion criteria to identify and select the eligible studies, and the methods to be used to extract and analyze the data (Egger \& Smith, 2001).

To check and guarantee the reliability in deciding about inclusion or exclusion of primary studies, it is useful to have at least two raters checking the eligibility of the primary studies and resolve any disagreements by discussion or through a third reviewer (Egger \& Smith, 2001). Well-conducted SRs/MAs need at least two independent persons for selecting eligible studies and extracting data to reduce potential errors, which one person or dependent persons may commit (Higgins \& Deeks, 2008; Jones, Remmington, Williamson, Ashby, \& Smyth, 2005). Despite this, only about one third of the studies in this review explicitly stated that at least two persons independently have checked the eligibility of the primary studies and selected them afterwards (Item 2). However, a considerable percentage of the studies mentioned that at least two independent coders extracted data from the primary studies.

Although Items 7 and 8 on the assessment of the quality of primary studies and accounting for the quality in drawing conclusions indicated some improvements over time, only around one third of the studies appraised and documented clearly the level of scientific quality of the primary studies and not many of them utilized the results of this scientific appraisal for formulating the conclusions and recommendations. Alarcón et al. (2017), Bao et al. (2014), Deckert et al. (2014), and Hall et al. (2017) in their reviews found that most of the included SRs and MAs did not take into account these domains. Validity and reliability of MAs are dependent on the quality of the primary included studies, so if low quality studies are aggregated, the overall effect may not be an accurate measure of the true population effect. Meta-analysts and reviewers can avoid this kind of inaccuracy by assessing the methodological quality of the included studies through validated tools. Afterwards, they must try to use the results of the studies' quality in formulating the conclusions and recommendations (Hall et al., 2017). 
Furthermore, it should be noted that except for Items 3 and 6 (on the comprehensiveness of the literature search and on reporting the characteristics of included studies) that got an overall score higher than 0.50 (in the theoretical range of 0-1), the remaining items did not even reach this middle point and most of them have a very low mean and median, although some of these items demonstrated some improvement over time. Poor quality primary studies included in MAs and SRs, heterogeneity between these studies, and possible publication biases can lead to biased results (Sygouros \& Acar, 2013). Since these items obtained low scores, it is crucial to consider them for conducting SR and MA.

None of the 44 criteria of the modified R-AMSTAR was fulfilled by all SCED MAs, which is similar to the findings of Alarcón et al. (2017), and two criteria ('study protocol or “a priori” design is published and/or registered in advance' [criterion 1A] and 'An awareness/statement of support or conflict of interest in the primary included studies' [criterion 11C]) were met by none of the studies, which was found also in the review of Alarcón et al. (2017). Some criteria of the 11 main items have been met by more SCED MAs. 'In an aggregated form such as a table, data from the original studies are provided on the participants, interventions/exposure, outcomes, ...' [criterion 6A], 'used databases are mentioned' [criterion 3C], 'at least two electronic sources are searched' [criterion 3A], 'at least two persons independently extracted the data, explicitly stated' [criterion 2D], and 'searches should be supplemented by consulting current contents, reviews, textbooks, the reference lists of eligible studies, citation indices, contacting experts' [criterion 3E] were met by $94.40 \%, 93.30 \%, 88.80 \%, 85.40 \%$, and $80.30 \%$ of the SCED MAs, respectively. Rotta et al. (2015) found similar results for some of these criteria $(6 \mathrm{~A}, 3 \mathrm{C}, \& 3 \mathrm{~A})$.

\subsection{Limitations}

The current review was based on a systematic literature search strategy, thereby ensuring comprehensiveness. Still, as many other studies, it has some limitations. The main limitation of current review is that the methodological quality of SCED MAs could only be evaluated based on what was reported explicitly in the study. If the author(s) did not report an action in the manuscript, the RAMSTAR score assigned reflects that the action was not performed. For example, if the author(s) searched for grey literature, but did not mention it explicitly in the manuscript, the 'no' score was assigned to this criterion. The methodological quality scores therefore may underestimate the actual quality of the SCED MAs.

We could not find any study that specifically assessed the methodological quality of SCED MAs/SRs through R-AMSTAR. Therefore, we could just compare some of our findings with the studies that applied this tool for quality assessment in other contexts, but not in contexts of MA of SCEDs.

Another limitation is that, as Hall et al. (2017) pointed out, the R-AMSTAR assigns the same weight to all criteria, but in fact some criteria may have a greater impact on the validity of the results than other criteria. As an example, evaluating the quality of the individual studies and applying the results in conclusions and formulating recommendation can affect the validity more than the inclusion of a list of included or excluded studies. Therefore, caution should be given to the interpretation of the total score. Even though a higher overall score of R-AMSTAR indicates a better methodological quality in comparison to lower score, there is not a definite cutoff value to judge quality. Regarding the fact that some of the MAs did not systematically select, extract, and aggregate the results of primary studies, it can lead to low methodological quality scores based on the R-AMSTAR items which have been developed mainly for SRs. Although Pieper et al. (2014) found that R-AMSTAR can be used for methodological quality assessment of nonrandomized MAs and SRs and we modified some criteria for our purpose, this modified R-AMSTAR is still not specifically developed and designed for SCED MAs. Therefore, it may not be fair to evaluate the quality of SCED MAs using criteria that are deemed important in MA of group comparison studies, e.g., the assessment of publication bias, mentioning the conflict of interest in primary studies, etc. All of the results in the current review therefore should be interpreted by caution, although we believe that the results from the R-AMSTAR provide at least a strong indication of methodological quality.

\subsection{Recommendations}

Due to the insufficient methodological quality found for the included SRs/MAs, it is necessary that future SCED meta-analysts and reviewers try to improve and optimize the scientific rigor of their work. Employing some checklists, such as R-AMSTAR, AMSTAR, OQAQ, PRISMA, ... in the early stages of study design can be helpful. As the findings of the current review illustrate, for most of the methodological aspects, we obtained low scores and improving these deficiencies may reduce misleading findings and increase the validity of results. Future SCED meta-analysts should focus on the following crucial methodological aspects:

- Publishing and registering a study protocol or 'a priori' design in advance in which the meta-analysts provide all details about the study procedures and develop the research questions/objectives based on the PICO format would be a useful guideline to manage a valid MA. In case the reviewers deviate (in one way or another) from this protocol while conducting their actual review, these deviations should be explicitly documented in their final review.

- More comprehensive literature search strategies for published and unpublished studies without restriction on the language (e.g. just English) can decrease publication bias. Statistical tests and graphical aids would be more informative to assess and report the likelihood of publication bias, because it is an important issue in performing MAs.

- The heterogeneity among primary studies must be checked and in the presence of heterogeneity, appropriate methods like a random effects model must be applied when statistically analyzing meta-analytic databases.

- Due to the influence of the quality of primary data on the validity of pooled results in SCED MAs, the scientific quality of primary studies must be assessed and documented in-depth through validated instruments. Afterwards, the meta-analysts should use these 
results in formulating the conclusions and recommendations.

- Some SCED MAs (Jitendra, Burgess, \& Gajria, 2011; Maggin, Chafouleas, Goddard, \& Johnson, 2011; Murray, McCabe, \& Ballard, 2014; Schlosser \& Wendt, 2008) had higher methodological quality scores compared to other studies in the current review according to modified R-AMSTAR. These studies could be useful as guides for SCED meta-analysts, although the quality of these studies could be improved as well.

Further research can be undertaken to investigate the methodological quality of the SCED MAs and SRs by applying other instruments and checklists. In future studies, it might be possible to use different checklists to assess the methodological quality of the current dataset and examine the agreement of the results of different assessments. It would be fruitful to develop and validate further a tool to evaluate specifically the methodological quality of SCED MAs and SRs.

\subsection{Conclusion}

Although SRs and MAs are powerful methods to summarize and synthesize data from primary studies, they are at risk of erroneous and biased methodology and reporting. The main findings of the current review demonstrate that the methodological quality of the SCED MAs has increased over the time, but should be improved further. SCED meta-analysts and systematic reviewers should make more efforts to reduce methodological flaws by considering higher methodological standards when conducting and reporting their reviews and increase the confidence in the validity of the results. The use of a standardized checklist (e.g., AMSTAR, R-AMSTAR, OQAQCASP, SIGN, PRISMA, etc.) can help in assessing the quality of SCED MAs, but can also help SCED meta-analysts to conduct a high methodological quality review of primary studies with minimal errors and biases.

\section{Conflict of interest}

Authors declare that they have no conflict of interest.

\section{Funding}

This project was supported in part by the Institute of Education Sciences, U.S. Department of Education, under Grant R305D150007. All the content is solely the responsibility of the authors and do not represent views of the Institute of Education Sciences, U.S. Department of Education.

\section{Acknowledgements}

All the authors are grateful to Emily Rodabaugh, Maries Vissenaeken, and Dimitri Vandersteen for their helpful contribution to the identification of relevant studies and to data extraction.

\section{References}

Alarcón, M., Diaz, K. T., Aranda, L., Cafferata, E. A., Faggion, C. M., \& Monje, A. (2017). Use of biologic agents to promote bone formation in implant dentistry: A critical assessment of systematic reviews. The International Journal of Oral \& Maxillofacial Implants, 32(2), 271-281. http://dx.doi.org/10.11607/jomi.5101.

Bao, Y., Kong, X., Yang, L., Liu, R., Shi, Z., Li, W., \& Hou, W. (2014). Complementary and alternative medicine for cancer pain: An overview of systematic reviews. Evidence-Based Complementary and Alternative Medicine, 2014. http://dx.doi.org/10.1155/2014/170396.

Barlow, D. H., Nock, M. K., \& Hersen, M. (2009). Single case experimental designs (3rd). Boston: Pearson/Allyn and Bacon.

Beretvas, S. N., \& Chung, H. (2008a). A review of meta-analyses of single-subject experimental designs: Methodological issues and practice. Evidence-Based Communication Assessment and Intervention, 2(3), 129-141. http://dx.doi.org/10.1080/17489530802446302.

Beretvas, S. N., \& Chung, H. (2008b). An evaluation of modified R 2-change effect size indices for single-subject experimental designs. Evidence-Based Communication Assessment and Intervention, 2(3), 120-128. http://dx.doi.org/10.1080/17489530802446328.

Borenstein, M., Hedges, L. V., Higgins, J. P. T., \& Rothstein, H. R. (2009). Introduction to meta-Analysis. Chichester: Wileyhttp://dx.doi.org/10.1002/9780470743386. fmatter.

Brossart, D. F., Meythaler, J. M., Parker, R. I., McNamara, J., \& Elliott, T. R. (2008). Advanced regression methods for single-case designs: Studying propranolol in the treatment for agitation associated with traumatic brain injury. Rehabilitation Psychology, 53(3), 357-369. http://dx.doi.org/10.1037/a0012973.

Burda, B. U., Holmer, H. K., \& Norris, S. L. (2016). Limitations of a measurement tool to assess systematic reviews (AMSTAR) and suggestions for improvement. Systematic Reviews, 5(58), http://dx.doi.org/10.1186/s13643-016-0237-1.

Bushman, B. J., \& Wang, M. C. (2009). Vote- counting procedures in meta-analysis. In H. Cooper, L. V. Hedges, \& J. C. Valentine (Eds.). Handbook of research synthesis and meta-analysis(2nd ed.). New York: Russell Sage Foundation.

Campbell, J. M. (2004). Statistical comparison of four effect sizes for single-subject designs. Behavior Modification, 28(2), 234-246. http://dx.doi.org/10.1177/ 0145445503259264.

Corry, M., While, A., Neenan, K., \& Smith, V. (2015). A systematic review of systematic reviews on interventions for caregivers of people with chronic conditions. Journal of Advanced Nursing, 71(4), 718-734. http://dx.doi.org/10.1111/jan.12523.

Critical Appraisal Skills Programme (2013). CASP systematic review checklist. [Retrieved from http://www.casp-uk.net/casp-tools-checklists].

De Vito, C., Manzoli, L., Marzuillo, C., Anastasi, D., Boccia, A., \& Villari, P. (2007). A systematic review evaluating the potential for bias and the methodological quality of meta-analyses in vaccinology. Vaccine, 25(52), 8794-8806. http://dx.doi.org/10.1016/j.vaccine.2007.10.034.

Deckert, S., Kopkow, C., \& Schmitt, J. (2014). Nonallergic comorbidities of atopic eczema: An overview of systematic reviews. Allergy: European Journal of Allergy and Clinical Immunology, 69(1), 37-45. http://dx.doi.org/10.1111/all.12246.

Egger, M., \& Smith, G. D. (2001). Principles of and procedures for systematic reviews. In M. Egger, G. D. Smith, \& D. G. Altman (Eds.). Systematic reviews in health care: Meta-analysis in context (pp. 23-42). (2nd ed.). London: BMJ books.

Faggion, C. M. J., \& Giannakopoulos, N. N. (2013). Critical appraisal of systematic reviews on the effect of a history of periodontitis on dental implant loss. Journal of 
Clinical Periodontology, 40(5), 542-552. http://dx.doi.org/10.1111/jcpe.12096.

Faggion, C. M. J. (2015). Critical appraisal of AMSTAR: Challenges, limitations, and potential solutions from the perspective of an assessor. BMC Medical Research Methodology, 15(63), http://dx.doi.org/10.1186/s12874-015-0062-6.

Farmer, J. L., Owens, C. M., Ferron, J. M., \& Allsopp, D. H. (2010). A methodological review of single-case meta-analyses. Denvor: Paper presented at the American Educational Research Association.

Hall, A. M., Lee, S., \& Zurakowski, D. (2017). Quality assessment of meta-analyses published in leading anesthesiology journals from 2005 to 2014 . Anesthesia \& Analgesia, 124(6), 2063-2067. http://dx.doi.org/10.1213/ANE.0000000000002074.

Hedges, L. V., \& Olkin, I. (1983). Regression models in research synthesis. The American Statistician, 37(2), 137-140.

Hedges, L. V., \& Olkin, I. (1985). Statistical methods for meta-analysis. California: Academic Press.

Higgins, J. P., \& Deeks, J. J. (2008). Selecting studies and collecting data. In J. P. Higgins, \& G. Sally (Eds.). Cochrane handbook for systematic reviews of interventions. England: JohnWiley \& Sons Ltd.

Horner, R. H., Carr, E. G., Halle, J., McGee, G., Odom, S., \& Wolery, M. (2005). The use of single-subject research to identify evidence-based practice in special education. Exceptional Children, 71(2), 165-179. http://dx.doi.org/10.1177/001440290507100203.

Jitendra, A. K., Burgess, C., \& Gajria, M. (2011). Cognitive strategy instruction for improving expository text comprehension of students with learning disabilities: The quality of evidence. Exceptional Children, 77(2), 135-159. http://dx.doi.org/10.1177/001440291107700201.

Jones, A. P., Remmington, T., Williamson, P. R., Ashby, D., \& Smyth, R. L. (2005). High prevalence but low impact of data extraction and reporting errors were found in Cochrane systematic reviews. Journal of Clinical Epidemiology, 58(7), 741-742. http://dx.doi.org/10.1016/j.jclinepi.2004.11.024.

Kowalczuk, M., Adamich, J., Simunovic, N., Farrokhyar, F., \& Ayeni, O. R. (2015). Methodological quality of systematic reviews addressing femoroacetabular impingement. Knee Surgery Sports Traumatology Arthroscopy, 23(9), 2583-2589. http://dx.doi.org/10.1007/s00167-014-3151-x.

Kratochwill, T. R., Hitchcock, J., Horner, R. H., Levin, J. R., Odom, S. L., Rindskopf, D. M., \& Shadish, W. R. (2010). Single-case design technical documentation. What works clearing house. [Retrieved from What Works Clearinghouse website: http://ies.ed.gov/ncee/wwc/pdf/wwc_scd.pdf].

Kung, J., Chiappelli, F., Cajulis, O. O., Avezova, R., Kossan, G., Chew, L., \& Maida, C. A. (2010). From systematic reviews to clinical recommendations for evidencebased health care: Validation of revised assessment of multiple systematic reviews (R-AMSTAR) for grading of clinical relevance. The Open Dentistry Journal, 4, 84-91. http://dx.doi.org/10.2174/1874210601004020084.

Li, L., Ying, J., Sun, T.-T., Yi, K., Tian, H.-L., Sun, R., \& Yang, K.-H. (2012). Overview of methodological quality of systematic reviews about gastric cancer risk and protective factors. Asian Pacific Journal of Cancer Prevention, 13(5), 2069-2079.

Machalicek, W., Sanford, A., Lang, R., Rispoli, M., Molfenter, N., \& Mbeseha, M. K. (2010). Literacy interventions for students with physical and developmental disabilities who use aided AAC devices: A systematic review. Journal of Developmental and Physical Disabilities, 22(3), 219-240. http://dx.doi.org/10.1007/s10882009-9175-3.

Maggin, D. M., Chafouleas, S. M., Goddard, K. M., \& Johnson, A. H. (2011). A systematic evaluation of token economies as a classroom management tool for students with challenging behavior. Journal of School Psychology, 49(5), 529-554. http://dx.doi.org/10.1016/j.jsp.2011.05.001.

Maggin, D. M., O'Keeffe, B. V., \& Johnson, A. H. (2011). A quantitative synthesis of methodology in the meta-analysis of single-subject research for students with disabilities: 1985-2009. Exceptionality: A Special Education Journal, 19(2), 109-135. http://dx.doi.org/10.1080/09362835.2011.565725.

Moeyaert, M., Ugille, M., Ferron, J. M., Beretvas, S. N., \& Van de Noortgate, W. (2014a). The influence of the design matrix on treatment effect estimates in the quantitative analyses of single-subject experimental design research. Behavior Modification, 38(5), 665-704. http://dx.doi.org/10.1177/0145445514535243.

Moeyaert, M., Ugille, M., Ferron, J. M., Beretvas, S. N., \& Van de Noortgate, W. (2014b). Three-level analysis of single-case experimental data: Empirical validation. The Journal of Experimental Education, 82(1), 1-21. http://dx.doi.org/10.1080/00220973.2012.745470.

Moher, D., Liberati, A., Tetzlaff, J., Altman, D. G., \& The PRISMA Group (2009). Preferred reporting items for systematic reviews and meta-analyses: The PRISMA statement. PLoS Medicine, 6(7), http://dx.doi.org/10.1371/journal.pmed.1000097.

Murray, E., McCabe, P., \& Ballard, K. J. (2014). A systematic review of treatment outcomes for children with childhood apraxia of speech. American Journal of SpeechLanguage Pathology, 23, 486-504. http://dx.doi.org/10.1044/2014.

O’Donnell, A., Anderson, P., Newbury-Birch, D., Schulte, B., Schmidt, C., Reimer, J., \& Kaner, E. (2014). The impact of brief alcohol interventions in primary healthcare: A systematic review of reviews. Alcohol and Alcoholism, 49(1), 66-78. http://dx.doi.org/10.1093/alcalc/agt170.

Onghena, P., \& Edgington, E. S. (2005). Customization of pain treatments: Single-case design and analysis. The Clinical Journal of Pain, 21(1), http://dx.doi.org/10. 1097/00002508-200501000-00007 [56-68-72].

Onghena, P. (2005). Single-case designs. In B. S. Everittt, \& D. C. Howell (Eds.). Encyclopedia of statistics in behavioral science (pp. 1850-1854). Chichester UK: John Wiley \& Sons.

Owens, C. M. (2011). Meta-analysis of single-case data: A monte carlo investigation of a three level model (Doctoral dissertation). [Retrieved from Graduate Thesis and Dissertation].

Oxman, A. D., \& Guyatt, G. H. (1991). Validation of an index of the quality of review articles. Journal of Clinical Epidemiology, 44(11), 1271-1278. http://dx.doi.org/ 10.1016/0895-4356(91)90160-B.

Parke, H. L., Epiphaniou, E., Pearce, G., Taylor, S. J. C., Sheikh, A., Griffiths, C. J., \& Pinnock, H. (2015). Self-management support interventions for stroke survivors: A systematic meta-review. PLoS ONE, 10(7), http://dx.doi.org/10.1371/journal.pone.0131448.

Pearce, G., Pinnock, H., Epiphaniou, E., Parke, H. L., Heavey, E., Griffiths, C. J., \& Taylor, S. J. C. (2015). Experiences of self-management support following a stroke: A meta-review of qualitative systematic reviews. PLoS ONE, 10(12), http://dx.doi.org/10.1371/journal.pone.0141803.

Petit-Bois, M., Baek, E. K., Van den Noortgate, W., Beretvas, S. N., \& Ferron, J. M. (2016). The consequences of modeling autocorrelation when synthesizing single-case studies using a three-level model. Behavior Research Methods, 48(2), 803-812. http://dx.doi.org/10.3758/s13428-015-0612-1.

Pieper, D., Mathes, T., \& Eikermann, M. (2014). Can AMSTAR also be applied to systematic reviews of non-randomized studies? BMC Research Notes, 7(609), http://dx. doi.org/10.1186/1756-0500-7-609.

Pinnock, H., Parke, H. L., Panagioti, M., Daines, L., Pearce, G., Epiphaniou, E., \& Taylor, S. J. C. (2017). Systematic meta-review of supported self-management for asthma: A healthcare perspective. BMC Medicine, 15(64), http://dx.doi.org/10.1186/s12916-017-0823-7.

Popovich, I., Windsor, B., Jordan, V., Showell, M., Shea, B., \& Farquhar, C. M. (2012). Methodological quality of systematic reviews in subfertility: A comparison of two different approaches. PLoS ONE, 7(12), http://dx.doi.org/10.1371/journal.pone.0050403.

Ramchandani, M., Siddiqui, M., Kanwar, R., Lakha, M., Phi, L., Giacomelli, L., \& Chiappelli, F. (2010). Proteomic signature of periodontal disease in pregnancy: Predictive validity for adverse outcomes. Bioinformation, 5(7), 300-303. http://dx.doi.org/10.6026/97320630005300.

Rave, A., \& Rumohr, E. (2016). Socioeconomic health inequalities, their variation across countries, welfare regimes and methodological concepts: A meta-analysis (protocol). [Retrieved from http://www.crd.york.ac.uk/PROSPERO/display_record.asp?ID = CRD42016035779].

Remschmidt, C., Wichmann, O., \& Harder, T. (2014). Methodological quality of systematic reviews on influenza vaccination. Vaccine, 32(15), 1678-1684. http://dx. doi.org/10.1016/j.vaccine.2014.01.060.

Rice, D. B., Shrier, I., Kloda, L. A., Benedetti, A., \& Thombs, B. D. (2016). Methodological quality of meta-analyses of the diagnostic accuracy of depression screening tools. Journal of Psychosomatic Research, 84, 84-92. http://dx.doi.org/10.1016/j.jpsychores.2016.03.013.

Rogers, L. A., \& Graham, S. (2008). A meta-analysis of single subject design writing intervention research. Journal of Educational Psychology, 100(4), 879-906. http:// dx.doi.org/10.1037/0022-0663.100.4.879.

Rotta, I., Salgado, T. M., Silva, M. L., Correr, C. J., \& Fernandez-Llimos, F. (2015). Effectiveness of clinical pharmacy services: An overview of systematic reviews (2000-2010). International Journal of Clinical Pharmacy, 37(5), 687-697. http://dx.doi.org/10.1007/s11096-015-0137-9.

Schlosser, R. W., \& Wendt, O. (2008). Effects on augmentative and alternative intervention on speech production in children with autism: A systematic review. American Journal of Speech-Language Pathology, 17, 212-230. http://dx.doi.org/10.1044/1058-0360(2008/021).

Schlosser, R. W., Lee, D. L., \& Wendt, O. (2008). Application of the percentage of non-overlapping data (PND) in systematic reviews and meta-analyses: A systematic review of reporting characteristics. Evidence-Based Communication Assessment \& Intervention, 2(3), 163-187. http://dx.doi.org/10.1080/17489530802505412. 
Schmitter, M., Sterzenbach, G., Faggion, C. M., \& Krastl, G. (2013). A flood tide of systematic reviews on endodontic posts: Methodological assessment using of RAMSTAR. Clinical Oral Investigations, 17(5), 1287-1294. http://dx.doi.org/10.1007/s00784-013-0945-z.

Scottish Intercollegiate Guidelines Network (2012). Methodology checklist 1: Systematic reviews and meta-analyses. Retrieved from http://www.sign.ac.uk/checklistsand-notes.html.

Shadish, W. R., \& Rindskopf, D. M. (2007). Methods for evidence-based practice: Quantitative synthesis of single-subject designs. New Direction for Evaluation, 113, 95-109. http://dx.doi.org/10.1002/ev.217.

Shadish, W. R., Hedges, L. V., \& Pustejovsky, J. E. (2014). Analysis and meta-analysis of single-case designs with a standardized mean difference statistic: A primer and applications. Journal of School Psychology, 52(2), 123-147. http://dx.doi.org/10.1016/j.jsp.2013.11.005.

Shadish, W. R., Hedges, L. V., Horner, R. H., \& Odom, S. L. (2015). The role of between-case effect size in conducting, interpreting, and summarizing single-case research (NCER 2015-002). National Center for Education Research, Institute of Education.

Shadish, W. R. (2014a). Analysis and meta-analysis of single-case designs: An introduction. Journal of School Psychology, 52(2), 109-122. http://dx.doi.org/10.1016/j. jsp.2013.11.009.

Shadish, W. R. (2014b). Statistical analyses of single-case designs: The shape of things to come. Current Directions in Psychological Science, 23(2), 139-146. http://dx. doi.org/10.1177/0963721414524773.

Sharif, M. O., Janjua-Sharif, F. N., Sharif, F. N. J., Ali, H., \& Ahmed, F. (2013). Systematic reviews explained: AMSTAR-how to tell the good from the bad and the ugly. Oral Health and Dental Management, 12(1), 9-16.

Shea, B., Dube, C., \& Mohr, D. (2001). Assessing the quality of reports of systematic reviews: the QUOROM statement compated to other tools. In M. Egger, G. D. Smith, \& D. G. Altman (Eds.). Systemati reviews in health care: Meta-analysis in context (pp. 122-139). (2nd ed.). London: BMJ books.

Shea, B. J., Grimshaw, J. M., Wells, G. A., Boers, M., Andersson, N., Hamel, C., \& Bouter, L. M. (2007). Development of AMSTAR: A measurement tool to assess the methodological quality of systematic reviews. BMC Medical Research Methodology, 7(1), 10. http://dx.doi.org/10.1186/1471-2288-7-10.

Shea, B. J., Hamel, C., Wells, G. A., Bouter, L. M., Kristjansson, E., Grimshaw, J., \& Boers, M. (2009). AMSTAR is a reliable and valid measurement tool to assess the methodological quality of systematic reviews. Journal of Clinical Epidemiology, 62(10), 1013-1020. http://dx.doi.org/10.1016/j.jclinepi.2008.10.009.

Smith, J. D. (2012). Single-case experimental designs: A systematic review of published research and current standards. Psychological Methods, 17(4), http://dx.doi. $\operatorname{org} / 10.1037 / \mathrm{a} 0029312$.

Sygouros, A., \& Acar, A. (2013). Evidence-based orthodontics: Appraisal of the methodologies of systematic reviews and meta-analyses in controversial areas of orthodontics. Journal of the World Federation of Orthodontists, 2(3), e117-e122. http://dx.doi.org/10.1016/j.ejwf.2013.05.004.

Thompson, C., Schabrun, S., Romero, R., Bialocerkowski, A., \& Marshall, P. (2016). Factors contributing to chronic ankle instability: A protocol for a systematic review of systematic reviews. Systematic Reviews, 5(94), http://dx.doi.org/10.1186/s13643-016-0275-8.

Tincani, M., \& De Mers, M. (2016). Meta-analysis of single-case research design studies on instructional pacing. Behavior Modification, 40(6), 799-824. http://dx.doi. org $/ 10.1177 / 0145445516643488$.

Van den Noortgate, W., \& Onghena, P. (2003a). Combining single-case experimental data using hierarchical linear models. School Psychology Quarterly, 18(3), 325-346. http://dx.doi.org/10.1521/scpq.18.3.325.22577.

Van den Noortgate, W., \& Onghena, P. (2003b). Hierarchical linear models for the quantitative integration of effect sizes in single-case research. Behavior Research Methods Instruments \& Computers, 35(1), 1-10. http://dx.doi.org/10.3758/BF03195492.

Van den Noortgate, W., \& Onghena, P. (2008). A multilevel meta-analysis of single-subject experimental design studies. Evidence-Based Communication Assessment and Intervention, 2(3), 142-151. http://dx.doi.org/10.1080/17489530802505362.

Wei, X., Wang, S., Li, J., Gao, J., Yu, J., Feng, M., \& Zhu, L. (2015). Complementary and alternative medicine for the management of cervical radiculopathy: An overview of systematic reviews. Evidence-Based Complementary and Alternative Medicine, 2015. http://dx.doi.org/10.1155/2015/793649.

Wells, C., Kolt, G. S., Marshall, P., Hill, B., \& Bialocerkowski, A. (2013). Effectiveness of pilates exercise in treating people with chronic low back pain: A systematic review of systematic reviews. BMC Medical Research Methodology, 13(7), http://dx.doi.org/10.1186/1471-2288-13-7.

Zeng, X., Zhang, Y., Kwong, J. S. W., Zhang, C., Li, S., Sun, F., \& Du, L. (2015). The methodological quality assessment tools for preclinical and clinical studies, systematic review and meta-analysis, and clinical practice guideline: A systematic review. Journal of Evidence-Based Medicine, 8(1), 2-10. http://dx.doi.org/10. $1111 /$ jebm. 12141 